\title{
Mejora del Rendimiento Académico Mediante la Aplicación de Metodologías de Trabajo en Equipo y Sistemas de Respuesta Inmediata en la Universidad China Improvement of Academic Performance Through the Application of Teamwork Methodologies and Classroom Response Systems at the Chinese University
}

\author{
Francisco Rodríguez-Sedano ${ }^{1}$, Miguel A. Conde-González ${ }^{2}$, Xiaochen Yang ${ }^{3}$, $\mathrm{Jia} \mathrm{Fu}^{3}$ \\ francisco.sedano@unileon.es,miguel.conde@unileon.es,3547303@qq.com, fujia1014@xtu.edu.cn \\ ${ }^{1}$ Departamento de Ingeniería Eléctrica y \\ de Sistemas y Automática \\ Universidad de León \\ León, España \\ ${ }^{2}$ Departamento de Ingenierías Mecánica, \\ Informática y Aeroespacial \\ Universidad de León \\ Léon, España \\ ${ }^{3}$ School of Public Administration \\ Xiangtan University \\ Xiangtan-Hunan, China
}

\begin{abstract}
Resumen- La apertura educativa de China a través de acuerdos académicos con diferentes instituciones de formación superior del resto del mundo, supone también la posibilidad de aplicar herramientas y metodologías bien conocidas en contextos en los que su aplicación no se llevaba a cabo hasta ahora. En este sentido el presente trabajo persigue aplicar diferentes metodologías y herramientas para la gestión del trabajo en equipo y sistemas de respuesta presencial. Para ello se lleva a cabo un estudio cuasi-experimental en la Escuela Mecánica de la Universidad de Xiangtang durante tres años. En dicho estudio se observa el rendimiento académico de los alumnos en dos asignaturas, una como grupo de control y otra como grupo experimental. El grupo de control sigue el proceso tradicional de enseñanza en la Universidad China y en el grupo experimental un profesor de intercambio español adapta herramientas al contexto chino para fomentar el trabajo en equipo e incrementar la participación de los alumnos en clase. Los resultados obtenidos muestran un incremento de la participación de los estudiantes y de su rendimiento académico en la asignatura donde se aplicaron las diferentes técnicas y herramientas.
\end{abstract}

Palabras clave: China; Trabajo en Equipo; Sistemas de respuesta inmediata; Herramientas web.

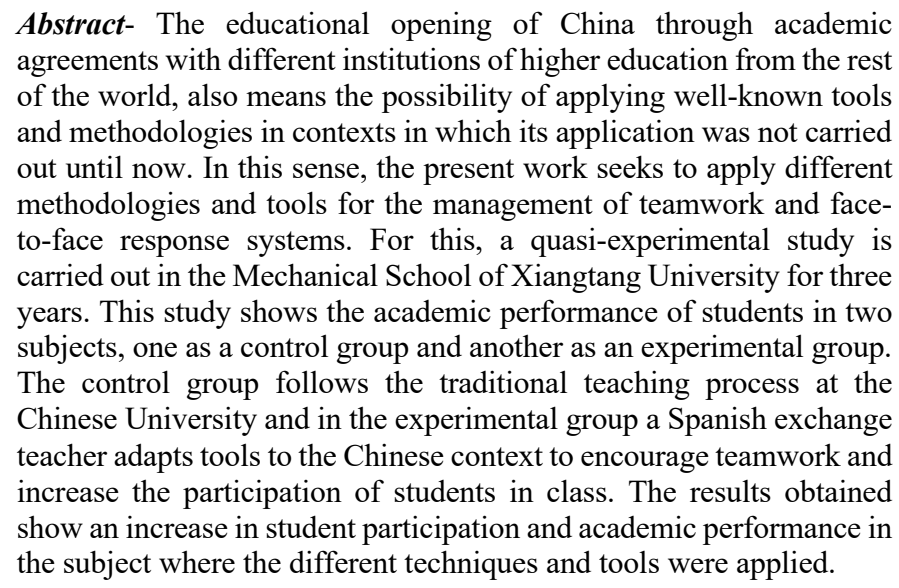

Abstract- The educational opening of China through academic agreements with different institutions of higher education from the rest of the world, also means the possibility of applying well-known tools and methodologies in contexts in which its application was not carried out until now. In this sense, the present work seeks to apply different methodologies and tools for the management of teamwork and faceto-face response systems. For this, a quasi-experimental study is carried out in the Mechanical School of Xiangtang University for three years. This study shows the academic performance of students in two subjects, one as a control group and another as an experimental group. The control group follows the traditional teaching process at the Chinese University and in the experimental group a Spanish exchange teacher adapts tools to the Chinese context to encourage teamwork and s obtained the subject where the different techniques and tools were applied.
Keywords: China; Teamwork; Classroom response systems; Web tools.

\section{INTRODUCCIÓN}

La educación, especialmente la educación superior, es un sector que en el que China se ha abierto al mundo en los últimos años. Según el Academic Ranking of World Universities (ARWU) ("Global Ranking of Academic Subjects," 2019), China tiene 3 universidades entre las 100 mejores y 51 universidades entre las mejores 500 del mundo, más que cualquier otro país asiático. La evolución en los últimos años ha sido espectacular, pasando de tener solo 32 universidades en este ranking en 2014, a 41 en 2016 y a las 51 universidades actuales. Hoy en día, las universidades chinas tienen programas de intercambio con más de 40 universidades de Estados Unidos, el Reino Unido, Australia, Canadá y otros países de Europa.

Debido a una política de reforma y apertura al mundo exterior en China, el número de estudiantes extranjeros que llegan a China se está incrementando constantemente. Las estadísticas del Ministerio de Educación revelan que este aumento se ha mantenido por encima del $20 \%$ desde el año 2000 hasta la actualidad (Ministry of Education, 2017). Este incremento se debe al hecho de que, aunque la educación completa es en chino, los estudiantes extranjeros tienen la oportunidad de estudiar otros programas en inglés (Khan, Waheed, Chengwen, Butt \& Ahmad, 2019). Más concretamente, la ingeniería en China se está volviendo muy popular entre los estudiantes internacionales y la cantidad de programas en este campo que se imparten en inglés es cada vez más amplia.

Además, en los últimos años, China ha capacitado a muchos ingenieros y técnicos calificados en una amplia gama de disciplinas (Guangya, 2008). Estos profesionales han desempeñado un papel importante en el desarrollo de la economía nacional, en la mejora continua del bienestar de las personas $\mathrm{y}$ en el avance de la ciencia y la tecnología, 
convirtiendo a China en el mayor productor de graduados en ingeniería del mundo. Así, el último informe anual (Engineering Fronts en 2018) realizado por el Centro de Estudios Estratégicos de la Chinese Academy of Engineering (CAE), y que incluye nueve campos de ingeniería, como la ingeniería mecánica, la ingeniería electrónica y de ciencias de la información y la ingeniería energética y minera, identifica 95 líneas de investigación y 96 líneas de desarrollo de ingeniería.

Por otro lado, hay que tener en cuenta que actualmente China se puede considerar como la segunda economía más grande del mundo después de Estados Unidos. La mayor parte de las 500 empresas más importantes del mundo están haciendo negocios en China (Liang, Yan, Quinlivan \& Cline, 2019), y muchas de estas grandes empresas han puesto en marcha sus plantas y líneas de producción y montaje en China (Wang, 2019). Esto ha desembocado en que muchas de estas compañías hayan establecido relaciones de cooperación con universidades chinas para absorber la gran cantidad de estudiantes extranjeros y chinos, para que realicen prácticas en sus empresas $\mathrm{y}$, posteriormente, emplearlos en las mismas. Muchos de estos estudiantes se colocaron en estos grupos de empresas internacionales después de graduarse en China, y algunos de ellos fueron contratados como gerentes en empresas subsidiarias en Estados Unidos, el Reino Unido y otros países europeos (Zhu \& Morgan, 2018).

También hay muchas posibilidades para que las empresas participen en proyectos de ingeniería llevados a cabo en el extranjero por el gobierno chino, a través de convenios bilaterales, como la construcción de infraestructuras y el desarrollo de nuevos recursos en países como Ghana, Sudáfrica y América Latina (Bersch \& Koivumaeki, 2019). Es precisamente la expansión en este mercado de habla hispana el que ha impulsado al gobierno chino para promover el aprendizaje del español en la educación superior y la materialización de acuerdos con diferentes universidades españolas (Ye \& Albornoz, 2018).

Al mismo tiempo, se puede afirmar que esta tendencia de las corporaciones multinacionales para acelerar su expansión en el mercado nacional chino ha generado grandes necesidades para los graduados en ingeniería con perfiles internacionales, especialmente aquellos que pueden hablar diferentes idiomas, incluido el chino. Para satisfacer estas necesidades, el gobierno chino ha promovido varias iniciativas, como programas de becas disponibles para estudiantes internacionales, como el programa de becas del Instituto Confucio y becas del gobierno local (Mrowiec-Denkowska, Chen, Zoll, \& Wang, 2019).

En este sentido, debe destacar el trabajo del Instituto Confucio en la educación y expansión de la cultura china en otros países. El Instituto Confucio es una nueva forma de cooperación educativa entre China y países extranjeros. El primer Instituto Confucio se estableció en 2004 en Seúl, Corea. Desde entonces, el número de convenios e institutos ha crecido rápidamente (Usmanova, Wang \& Younas, 2019). Según el último informe publicado por la Confucious Institute Headquarters (Haban, 2018), a finales de 2018, se habían establecido 548 Institutos Confucio, 1.193 Aulas Confucio y 5.665 centros de enseñanza en 154 países diferentes.

En resumen, aprovechando la apertura educativa del gobierno chino a través de convenios académicos con diferentes instituciones de formación superior en España y la labor del Instituto Confucio para favorecer estos acuerdos de cooperación educativa, se abre un nuevo horizonte que permite aplicar herramientas y metodologías bien conocidas en contextos educativos en los que su aplicación supone un cambio del enfoque tradicional del proceso de enseñanza-aprendizaje. Este es el objetivo principal de este trabajo, en el que se aplican dos metodologías diferentes, como son la gestión del trabajo en equipo y la utilización de sistemas de respuesta presencial, en un nivel educativo de enseñanza universitaria.

El resto de este trabajo está estructurado de la siguiente manera: la siguiente sección describe el contexto en el que se realiza el estudio. La Sección 3 presenta los materiales y métodos utilizados para la implementación de las nuevas metodologías en las asignaturas elegidas con el objetivo de mejorar el rendimiento académico de los estudiantes. La sección 4 muestra los principales hallazgos del experimento. En la sección 5 presentamos las lecciones aprendidas durante el estudio de caso presentado en este artículo que se analizan en la última sección.

\section{Contexto}

El contexto en el que se desarrolla el estudio se establece en diciembre de 2012, cuando el Ministerio de Educación de China aprobó su carrera de Diseño Mecánico, Fabricación y Automatización como un programa educativo cooperativo de grado, lo que lo convierte en el primer proyecto de cooperación en España para universidades chinas. El programa tiene dos modalidades diferentes. En la primera, 20 estudiantes de la Universidad de Xiangtan estudian de forma presencial dos cursos académicos en la Universidad de León. En la segunda modalidad, 9 profesores de la Universidad de León viajan a la Escuela de Ingeniería Mecánica en la Universidad de Xiangtan para impartir varias materias de los dos últimos cursos del grado durante cada año académico desde que comenzó el convenio.

Durante estos años, se ha observado que el mayor problema que tienen los estudiantes chinos es el idioma, ya que tanto las clases que reciben en la Universidad de León como las impartidas por los profesores en la Universidad de Xiangtan son en español. A lo largo de los dos primeros cursos del programa, todos los estudiantes estudian el idioma español, pero el nivel alcanzado no parece suficiente para garantizar la comprensión de algunos conceptos básicos de la ingeniería mecánica, por lo que los resultados de aprendizaje no son tan buenos como cabría esperar.

Para mejorar estos resultados, se ha llevado a cabo, durante tres cursos académicos consecutivos, la implementación de nuevas metodologías en la enseñanza de una asignatura impartida en la Universidad de Xiangtan por el mismo profesor de la Universidad de León. Estos resultados han sido comparados con los resultados de años anteriores y con otra materia del mismo semestre que se imparte con metodologías tradicionales, para que los alumnos del estudio sean los mismos y se puedan comparar su rendimiento académico.

A continuación, se describen las actividades desarrolladas en el estudio y los recursos utilizados.

\section{MATERIALES Y MÉTODOS}

El estudio se realizó en un diseño cuasi-experimental, sobre todo, debido al hecho de que no es logísticamente viable llevar a cabo un ensayo controlado aleatorio debido a las características de los sujetos del estudio que, en este caso, son 
estudiantes chinos de tercer y cuarto curso del grado de Diseño Mecánico, Fabricación y Automatización. Este diseño se usa a menudo para evaluar los beneficios de intervenciones específicas (Harris et al., 2006), cuando dicha intervención precede a la medición del resultado esperado. En este caso, la intervención consiste en la implementación de nuevas metodologías de enseñanza-aprendizaje.

Algunos autores clasifican los estudios cuasi-experimentales en cuatro categorías: diseños cuasi-experimentales sin grupos de control, diseños que usan grupos de control pero, sin prueba previa, diseños que usan grupos de control y pruebas preliminares y diseños de series de tiempo interrumpido (Shadish, Cook \& Campbell, 2002; Kampenes, Dybå, Hannay \& Sjøberg, 2009) . Este estudio de caso encaja en la última categoría; como observaciones espaciadas en intervalos de tiempo iguales, en este caso particular, durante el segundo semestre en años académicos consecutivos. La ventaja de este diseño es que, con múltiples mediciones tanto antes como después de la intervención, es más fácil corregir las posibles desviaciones del resultado deseable. Otra ventaja es que este diseño también puede mejorarse aún más incorporando muchas de las características de diseño mencionadas anteriormente en otras categorías, como la utilización de un grupo de control.

\section{A. Asignaturas y estudiantes objeto del estudio}

Las asignaturas elegidas para este estudio de caso fueron Tecnologías de Fabricación Avanzadas (TFA) e Introducción a la Ciencia y Tecnología Españolas (CTE). La elección de las mismas se realizó considerando varios factores clave. El primer fue que, durante los últimos tres cursos académicos, ambas asignaturas fueron impartidas por los mismos profesores de la Universidad de León. Otro factor influyente es que estos profesores tienen una amplia experiencia en la enseñanza universitaria. (22 y 28 años, respectivamente), así como en el uso de nuevas tecnologías aplicadas a la enseñanza en el campo de la ingeniería. Y el último factor clave es que las dos asignaturas se imparten en el mismo período de tiempo, dentro del segundo semestre del año académico.

Para este estudio, los alumnos seleccionados fueron de tercer curso, aunque en el caso de la asignatura CTE también hubo alumnos de cuarto año, ya que se trata de una asignatura optativa. Es por esta razón que los estudiantes de esta última asignatura fueran elegidos como un grupo de control. Además, esta asignatura se imparte siguiendo el método convencional basado en conferencias o clases magistrales.

Como se ha mencionado en el apartado anterior, todos los estudiantes objeto de este caso de estudio han realizado un curso de español durante los dos primeros años de grado. El objetivo es la adquisición de un nivel homogéneo de conocimientos del idioma español, idioma en el que los estudiantes reciben su docencia y que no es su lengua materna. Pero en años anteriores, los profesores se han quejado de que este nivel es demasiado bajo para entender algunos conceptos básicos de la ingeniería mecánica. Este hecho significa que los estudiantes no participan activamente en las clases y que los resultados obtenidos en la evaluación final de las asignaturas y su rendimiento académico no son los esperados.

Además, el sistema de evaluación es el tradicional en la educación superior en las universidades chinas, que consiste en un examen final y no es posible cambiarlo, lo que también supone un problema a la hora de introducir nuevas metodologías de enseñanza-aprendizaje. Es por esta razón, y con el objetivo de mejorar el rendimiento académico de los estudiantes, por lo que se decidió tratar de aprovechar las experiencias de varios de los profesores de la Universidad de León que participan en el convenio educativo para implementar progresivamente estas nuevas estrategias de enseñanzaaprendizaje. Estas metodologías se explican a continuación.

\section{B. Metodologías y herramientas utilizadas}

Muchos profesores de educación superior utilizan la metodología de trabajo en equipo para mejorar el aprendizaje de sus alumnos. Normalmente, el propósito es aumentar la comprensión del contenido por parte de los estudiantes (Curşeu \& Pluut, 2013), aunque esta metodología también se utiliza generalmente para desarrollar otras habilidades (Fransen, Kirschner \& Erkens, 2011). Este tipo de trabajo en grupo se llama aprendizaje colaborativo y se define como el uso educativo de pequeños grupos para promover que los estudiantes que trabajan juntos maximicen su propio aprendizaje y el de cada miembro del grupo (Johnson \& Johnson, 2008).

En este estudio se decidió utilizar un enfoque formal del aprendizaje cooperativo, donde los alumnos trabajan juntos durante uno o más períodos de clase para completar una tarea conjunta (Johnson, Johnson \& Smith, 2014). La tarea, en este caso concreto consistió en el análisis de varios artículos de revistas de investigación seleccionadas por el profesor y relacionadas con los conceptos teóricos de la asignatura TFA.

En este tipo de aprendizaje cooperativo, el profesor define los objetivos de aprendizaje de la actividad a realizar y asigna a los estudiantes a un grupo. Los expertos recomiendan usar grupos pequeños (de 2 a 6 estudiantes) y asignar roles a los miembros del grupo (Johnson, Johnson \& Smith, 2006). En el caso de la asignatura elegida para nuestro estudio, y dado que el número de alumnos varía entre 21 y 27 (según el año académico), se formaron grupos de 4 ó 5 alumnos.

Como herramienta de seguimiento, se utiliza un foro de discusión para observar el progreso del trabajo en equipo y donde los estudiantes participan de forma activa comentando el desarrollo y realización de la tarea encomendada por el profesor. Además, cada grupo debe hacer una exposición en clase una vez finalizada la tarea.

Como se discutió en la sección anterior, se decidió implementar estas metodologías de manera progresiva, por lo que esta metodología de trabajo en equipo se lanzó durante el primer año académico del experimento (2016/2017) y se mantuvo en los siguientes cursos (ver figura 1).

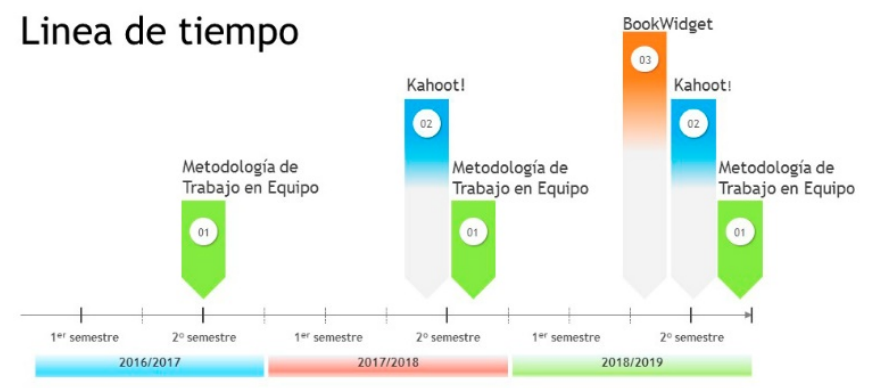

Figura 1: Cronología de la implementación de las diferentes metodologías durante los tres años académicos. 
Por otra parte, varios autores han demostrado que el uso de recursos basados en las nuevas tecnologías de la información y la comunicación llamados Sistemas de Respuesta Inmediata (SRI) en los procesos de enseñanza-aprendizaje en educación superior, mejora el rendimiento académico de los estudiantes en varios aspectos. Estos van, desde la participación debates o discusiones en clase (Hoffman \& Goodwin, 2006); la motivación, aprendizaje activo y la mayor asistencia a clase (Lucke, Dunn \& Christie, 2017); la preferencia del uso de estos sistemas para la participación de los estudiantes en las discusiones planteadas por el profesor, debido al anonimato de los participantes (Latham \& Hill, 2014); y la efectividad en el aprendizaje de conocimientos de tipo conceptual $o$ procedimental (Tregonning, Doherty, Hornbuckle \& Dickinson, 2012).

En este estudio de caso, los SRI empleados fueron Kahoot! y BookWidgets. A continuación, se describen con más detalle estas herramientas software y su funcionalidad.

Kahoot! Es una plataforma de aprendizaje basada en juegos, que permite al profesor administrar exámenes, discusiones o encuestas. Este software permite ser utilizado por toda la clase en tiempo real. Las preguntas, que suelen ser de opción múltiple, planteadas por el profesor, son proyectadas en la pantalla y los estudiantes responden a las preguntas con su teléfono inteligente (Smartphone), Tablet u ordenador portátil. En este estudio de caso, se realizan varias pruebas al final de cada lección teórica (un total de 8 temas). Al final del curso, también se lleva a cabo un concurso con todas las lecciones vistas en el tema. El objetivo es fortalecer los conceptos teóricos vistos en clase antes de la prueba escrita final, haciéndolo de un modo atractivo para el estudiante y motivando su participación. Este método se implementó durante el segundo curso académico (2017/2018) y en el último (2018/2019).

BookWidget es una plataforma para profesores que permite crear ejercicios interactivos y evaluar automáticamente las calificaciones, asignarlas a los estudiantes y darles una opinión o realimentación constructiva. Con esta herramienta, se diseñan varios ejercicios interactivos para que los estudiantes puedan relacionar los diferentes conceptos teóricos vistos en los temas de la materia y se puedan aplicar para resolver un problema planteado por el profesor. En total, se plantearon 4 ejercicios de este tipo durante el período en el que se impartió la asignatura. Esta herramienta se implementó en este último año académico (2018/2019).

Los resultados del estudio de caso se describirán a continuación.

\section{Resultados}

Para evaluar a los estudiantes que han participado en este estudio, es necesario adaptarse a los estándares de la Universidad de Xiangtan. De acuerdo con estas normas, para calcular la nota final de la asignatura, esta se debe adaptar al sistema de calificación de 100 puntos y se requerirán 60 puntos para obtener aprobar la asignatura. La nota final se puede dividir en dos partes; por una parte, la nota del examen final escrito, que representa el $70 \%$ de la calificación final; y por otro lado la nota de asistencia a clase, que representa el 30\% restante de la nota final.

Para evaluar los resultados de la implementación de las metodologías de este estudio de caso, se debe tener en cuenta que la primera metodología debe reflejarse en la calificación de asistencia a clase, que como se explicó anteriormente, representa el $30 \%$ de la calificación final. Hasta ahora, solo se consideraba la asistencia y participación en el aula del alumno. Pero en este caso de estudio, se añade la evaluación de la tarea planteada por el profesor y que se realiza en equipo. Además, también se evalúa la exposición que cada grupo hace en clase, una vez completada la tarea.

La implementación de las otras dos herramientas en los cursos posteriores, también debe reflejar una mejora en los resultados de los estudiantes también en este porcentaje $(30 \%$ de la nota final), ya que fomentan la participación activa de los estudiantes en clase. Pero también se espera que mejoren los resultados en el final examen final escrito ( $70 \%$ de la nota final), ya que la utilización de estos SRI, permiten al estudiante mejorar en la comprensión de los conocimientos de tipo conceptual o procedimental que se evalúan en dicho examen escrito.

Si se analizan estos resultados, se puede observar cómo se mejora el rendimiento de los estudiantes en la asignatura de TFA, donde se implementan las nuevas metodologías, como puede ver en la Tabla 1. En esta tabla, solo se compara el resultado de la calificación de la nota de asistencia a clase, que, como se ha indicado anteriormente, representa el $30 \%$ de la nota final. Además, como puede ver en los resultados de esta asignatura, el porcentaje de estudiantes aprobados mejora cada curso y aumenta el promedio. Comparando estos resultados con la asignatura de control (CTE), los resultados son mejores en la asignatura TFA en todos los cursos académicos. La nota media también mejora en el caso de la asignatura TFA, siendo la desviación estándar menor que en el caso la asignatura CTE.

\section{Tabla 1}

Resultados de la evaluación de la asistencia a clase (30\%).

\begin{tabular}{|r|cc|ccc|cc|}
\multicolumn{1}{c}{} & \multicolumn{2}{c}{$2016 / 2017$} & \multicolumn{2}{c}{$2017 / 2018$} & \multicolumn{2}{c|}{$2018 / 2019$} \\
\cline { 2 - 8 } & TFA & CTE & \multicolumn{1}{c|}{ TFA } & CTE & TFA & CTE \\
\hline $\begin{array}{r}\text { Tasa de alumnos } \\
\text { aprobados }\end{array}$ & $62 \%$ & $30 \%$ & $76 \%$ & $45 \%$ & $78 \%$ & $46 \%$ \\
\hline Media & 71 & 52 & 78 & 68 & 76 & 71 \\
\hline $\begin{array}{r}\text { Desviación } \\
\text { estándar }\end{array}$ & 8,73 & 11,60 & 7,68 & 8,00 & 6,67 & 9,14 \\
\hline $\begin{array}{r}\text { Número de } \\
\text { estudiantes }\end{array}$ & \multicolumn{2}{|c|}{27} & \multicolumn{2}{|c|}{21} & \multicolumn{2}{|c|}{23} \\
\hline
\end{tabular}

Si ahora se hace un análisis de la calificación final (ver Tabla 2), se puede concluir que, en la asignatura TFA, donde las nuevas metodologías se aplican progresivamente, la tasa de estudiantes aprobados también ha aumentado progresivamente. Más concretamente, $12,7 \%$ entre los cursos académicos $2016 / 17$ y $2017 / 18$ y $22,5 \%$ entre los cursos 2017/18 y 2018/19.

Tabla 2

Resultados de la evaluación final.

\begin{tabular}{|rcc|cc|cc|}
\multicolumn{1}{c}{} & \multicolumn{2}{c}{$2016 / 2017$} & $2017 / 2018$ & \multicolumn{2}{c|}{$2018 / 2019$} \\
\cline { 2 - 7 } \multicolumn{1}{c|}{} & TFA & CTE & TFA & CTE & TFA & CTE \\
\hline $\begin{array}{r}\text { Tasa de alumnos } \\
\text { aprobados }\end{array}$ & $63 \%$ & $48 \%$ & $71 \%$ & $52 \%$ & $87 \%$ & $52 \%$ \\
\hline $\begin{array}{r}\text { Media } \\
\text { Desviación } \\
\text { estándar }\end{array}$ & 64 & 53 & 71 & 63 & 82 & 71 \\
\hline 26,87 & 21,51 & 18,95 & 21,9 & 13,74 & 15,43 \\
\hline
\end{tabular}




\begin{tabular}{|r|l|l|l|}
\hline $\begin{array}{l}\text { Número de } \\
\text { estudiantes }\end{array}$ & 27 & 21 & 23 \\
\hline
\end{tabular}

También se puede ver en la tabla 2 que el rendimiento académico de los estudiantes ha mejorado en la asignatura de control (CTE), aunque en menor medida. Esto es lógico, ya que cuanto mejor es el estudiante, mayor es su rendimiento académico en cualquier asignatura (Farooq, Chaudhry, Shafiq \& Berhanu, 2011). Por lo tanto, será obvio que los estudiantes que obtienen buenas calificaciones en la asignatura de TFA tendrán también buenos resultados en la asignatura de CTE, como puede ver en la tabla al analizar la nota media.

\section{LECCIONES APRENDIDAS Y RECOMENDACIONES.}

Las lecciones aprendidas durante el estudio de caso presentado en este artículo pueden se pueden analizar desde diferentes puntos de vista. Por un lado, los autores creen que los SRI se pueden considerar una buena herramienta en el contexto en el que se desarrolla es estudio, puesto que permiten recopilar el conocimiento de los estudiantes sobre varios aspectos y conceptos relacionados con la asignatura en la que se utilizan, con una garantía de respuestas anónimas destinadas a evaluar el nivel real de conocimientos de los estudiantes y compararlo con su propia percepción de adquisición de dichos conocimientos.

Por otro lado, la participación activa de los estudiantes en clase y en los temas de discusión planteados por el profesor también ha mejorado en la asignatura TFA con el uso de estos sistemas, comparándolos con la participación en la asignatura de control (CTE).

Por último, el hecho de enfrentarse a la realización de una tarea en equipo en lugar de hacerlo de forma individual, ha representado para los estudiantes un cambio importante de mentalidad. Además, al tener que hacer una exposición oral al final de la tarea, lejos de representar un problema, ha sido una motivación adicional en la mayoría de los casos. Quizás el mayor problema para algunos estudiantes ha sido la expresión oral en un idioma que no es el suyo propio. Sin embargo, al utilizar las técnicas de trabajo en grupo mencionadas anteriormente, son los propios estudiantes los que ayudan a sus compañeros a superar estos problemas y miedos a la hora de exponer los resultados de su trabajo.

\section{CONCLUSIONES}

El estudio presentado en este artículo surge como resultado de la experiencia previa de varios profesores de la Universidad de León que han participado en un acuerdo académico firmado con la Universidad de Xiangtan, China. Son precisamente estos profesores los que llevarán a cabo este estudio que surge de la necesidad de mejorar el rendimiento académico de los estudiantes que participan en el programa educativo del que es objeto dicho convenio de cooperación entre ambas universidades. Dichos docentes cuentan con una amplia experiencia docente en educación superior, y más concretamente, en el campo de la ingeniería y en el uso de las nuevas tecnologías de la información y la comunicación en este campo.

Para este estudio de caso, se seleccionan dos asignaturas del segundo semestre que se impartirán durante tres cursos académicos consecutivos. La muestra se realiza con los mismos alumnos en estas dos asignaturas (en total participan en el caso de estudio 71 alumnos), seleccionando a los estudiantes de una de las asignaturas como grupo de control. En una de las asignaturas se implementaron diferentes metodologías de forma progresiva durante estos tres cursos académicos. La primera metodología que se implementa es la metodología de trabajo en equipo. Esta metodología ha representado un cambio importante en la mentalidad de los estudiantes, ya que es la primera vez que realizan una tarea grupal.

En los siguientes cursos académicos, se implementaron metodologías para incentivar la participación activa de los estudiantes en el aula, con herramientas conocidas como sistemas de respuesta inmediata en el aula. Para este fin, se eligen dos plataformas bien conocidas, que permiten al profesor diseñar diferentes actividades que motivan a los estudiantes a participar en el proceso de enseñanza-aprendizaje. Además, permiten al profesor asegurarse de que los estudiantes entiendan ciertos conceptos y que puedan razonar y procesar esta información para, posteriormente, aplicar estos conocimientos en los ejercicios y tareas planteadas en la asignatura en el que se ha implementado previamente la metodología de trabajo en equipo.

En resumen, este estudio contribuye a demonstrar que las dos metodologías implementadas en la asignatura elegida, contribuyen a mejorar los resultados de los estudiantes en comparación con los resultados obtenidos por los mismos estudiantes en la asignatura de control.

Los autores creen que el análisis y los resultados del estudio podrían mejorarse utilizando, por ejemplo, técnicas de análisis analítico (learning analytics). Pero para hacerlo, el número de estudiantes en la muestra tendría que aumentar significativamente. El número de asignaturas en las que se implementan las nuevas metodologías de aprendizaje también podría incrementarse. Los autores creen que esto revelaría que al aumentar el rendimiento académico del estudiante en una materia, esto influye en las otras asignaturas del grado.

\section{AgRADECIMIENTOS}

Esta sección es optativa.

\section{REFERENCIAS}

Bersch, K. \& Koivumaeki, R. I. (2019). Making Inroads: Infrastructure, State Capacity, and Chinese Dominance in Latin American Development. Studies in Comparative International Development, 1-23.

Confucius Institute Headquarters (Haban) (2018). Recuperado de http://www.hanban.org/report/2018.pdf

Curşeu, P. L., \& Pluut, H. (2013). Student groups as learning entities: The effect of group diversity and teamwork quality on groups' cognitive complexity. Studies in Higher Education, 38(1), 87-103.

Farooq, M. S., Chaudhry, A. H., Shafiq, M., \& Berhanu, G. (2011). Factors affecting students' quality of academic performance: a case of secondary school level. Journal of quality and technology management, 7(2), 1-14.

Fransen, J., Kirschner, P. A., \& Erkens, G. (2011). Mediating team effectiveness in the context of collaborative learning: The importance of team and task awareness. Computers in Human Behavior, 27(3), 1103-1113. 
Global Ranking of Academic Subjects (2019). Recuperado de http://www.shanghairanking.com/

Guangya, Z. (2008). Engineering in China (editorial). The Bridge, 28 (2), 2-4.

Harris, A. D., McGregor, J. C., Perencevich, E. N., Furuno, J. P., Zhu, J., Peterson, D. E., \& Finkelstein, J. (2006). The use and interpretation of quasi-experimental studies in medical informatics. Journal of the American Medical Informatics Association, 13(1), 16-23.

Hoffman, C., \& Goodwin, S. (2006). A clicker for your thoughts: Technology for active learning. New Library World, 107(9/10), 422-433.

Johnson, D. W., Johnson, R. T., \& Smith, K. A. (2006). Active learning: Cooperation in the university classroom $\left(3^{\text {rd }}\right.$ edition). Edina, MN: Interaction.

Johnson, D. W., Johnson, R. T., \& Smith, K. A. (2014). Cooperative learning: Improving university instruction by basing practice on validated theory. Journal on Excellence in University Teaching, 25(4), 1-26.

Johnson, R. T., \& Johnson, D. W. (2008). Active learning: Cooperation in the classroom. The annual report of educational psychology in Japan, 47, 29-30.

Kampenes, V. B., Dybå, T., Hannay, J. E., \& Sjøberg, D. I. (2009). A systematic review of quasi-experiments in software engineering. Information and Software Technology, 51(1), 71-82.

Khan, N.M., Waheed, M., Chengwen, H., Butt, T. M. \& Ahmad, J. (2019). Development and Perspectives of English, as a Language of Instruction and Learning in Chinese Educational System. Asian Journal of Contemporary Education, 3(1), 28-35.

Latham, A., \& Hill, N. S. (2014). Preference for anonymous classroom participation: Linking student characteristics and reactions to electronic response systems. Journal of Management Education, 38(2), 192-215.

Liang, B., Yan, L., Quinlivan, G. \& Cline, T. W. (2019). China's way to the US market: China's outward direct investment in the United States. In Handbook on China and Globalization. Edward Elgar Publishing.
Lucke, T., Dunn, P. K., \& Christie, M. (2017). Activating learning in engineering education using ICT and the concept of 'Flipping the classroom'. European Journal of Engineering Education, 42(1), 45-57.

Ministry of Education of the People's Republic of China (2017). Educational Statistics in 2017. Recuperado de http://en.moe.gov.cn/Resources/Statistics/edu_stat2017/n ational/

Mrowiec-Denkowska, J., Chen, Y., Zoll, F. S., \& Wang, K. (2019). Challenges Facing Chinese and European Universities in Mobility Cooperation: Managerial Administrative Staff Perspectives. In University Governance and Academic Leadership in the EU and China, 313-324. IGI Global.

Shadish, W. R., Cook, T. D., \& Campbell, D. T. (2002). Experimental and quasi-experimental designs for generalized causal inference.

Tregonning, A. M., Doherty, D. A., Hornbuckle, J., \& Dickinson, J. E. (2012). The audience response system and knowledge gain: a prospective study. Medical teacher, 34(4), e269-e274.

Usmanova, K., Wang, D., \& Younas, A. (2019). Brand Promotion of Confucius Institute in China from the Perspective of Cultural Recipients. In Proceedings of the 2019 3rd International Conference on Management Engineering, Software Engineering and Service Sciences, 102-106. ACM.

Wang, H. (2019). China's outward investment: Chinese enterprise globalization's characteristics, trends and challenges. In Handbook on China and Globalization. Edward Elgar Publishing.

Ye, P. \& Albornoz, L. A. (2018). Chinese Media 'Going Out'in Spanish Speaking Countries: The Case of CGTNEspañol. Westminster Papers in Communication and Culture, 13(1).

Zhu, J. \& Morgan, G. (2018). Global supply chains, institutional constraints and firm level adaptations: A comparative study of Chinese service outsourcing firms. Human Relations, 71(4), 510-535. 